\title{
To the question of the molecular structure of humic acids of soil humus
}

Bezuglova O.

Southern Federal University, Rostov-on-Don, Russia, lola314@mail.ru

doi: 10.36291/HIT.2019.bezuglova.022

There are still no clear molecular formulas for humic substances (HSs), all the suggested options have a schematic hypothetical character. In the second half of the 20th century, the concept of HSs of different origin was developed: they have a common principle of structure with a nucleus composed of an aromatic carbon skeleton substituted by alkyl and functional groups and a peripheral part enriched with polysaccharide and polypeptide fragments. However, some researchers believed that the HA molecule does not contain repetitive subunits and consists of randomized polymers, including various aromatic fragments. This concept is consistent with the hypothesis advanced by Piccolo [1], who suggested that HSs have a supramolecular structure. The supramolecular nature of humus acids could be considered proven by a number of instrumental techniques.

Of interest are studies focusing on the structural organization and parameters of these structures in soils of different genesis and in different soil horizons. The objects of our study were HAs (FAs) extracted from soils in Rostov region. The X-ray structural analysis of HAs was conducted using X-ray.

It has been established that the supramolecular organization of HAs in Chernozems and Chestnut soils can be described as a spatial structure composed of 2-4 layers of condensed aromatic systems supplemented by a network of chain fragments with different degrees of regularity and lengths. Structural parameters vary among soils of different genesis and genetic horizons of the same soil. Interplanar distances are distinctly registered; they vary from 0.349 to $0.371 \mathrm{~nm}$. Down the profile an increase in the interplanar distances and a decrease in the thickness of sets of chain fragments are observed. It seems reasonable to assume that these primary particles of HSs consist of formations that represent spatial structures of several layers of condensed aromatic systems supplemented by a network comprised of chain fragments with different degrees of regularity and lengths, as is schematically represented in Figure.

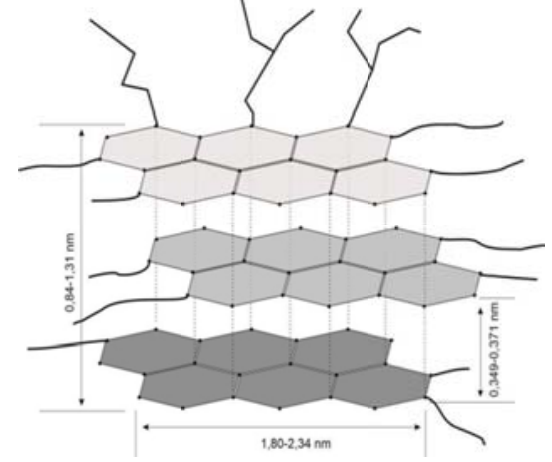

Figure. Hypothetical structure of a humic acid structural fragment

Thus, our results suggest that humus compounds are organized as several oligomer molecules associated into more or less condensed systems forming the basis for mobile and steady supramolecular formations.

Acknowledgement. This research was supported by the Russian Federation Leading Scientific School (NSH-3464.2018.11).

References

1. Piccolo A. // Soil Sci. 2001. 166(11):810-832. 